REF - ISSN 1808-0804 Vol. VI (2), 72 - 84, 2009

\title{
DESENVOLVIMENTO E VALIDAÇÃO DE MÉTODO ANALÍTICO ESPECTROFOTOMÉTRICO PARA DOSEAMENTO DE FUROSEMIDA MATÉRIA-PRIMA
}

Developed and validation of spectrophotometrical analytical method for quantification of furosemide raw material

\section{Lívia Teixeira Duarte; Ezequiane Machado Silva; Cláudia Gomes Miranda; Emilia Ferreira Lopes; Hanna Lopes Morais; Izabella Lobo; Clévia Ferreira Duarte Garrote; Maria Teresa Freitas Bara*} Laboratório de Controle de Qualidade de Medicamentos (LCQM), Faculdade de Farmácia; Universidade Federal de Goiás

*Autor para correspondência e-mail: mbara@farmacia.ufg.br

\section{Recebido em 18/06/2008 - Aceito em 11/02/2009}

\section{RESUMO:}

Furosemida é um fármaco que atua como diurético empregado no tratamento de hipertensão leve a moderada. Dentre as metodologias utilizadas para sua determinação, destaca-se a possibilidade de quantificação da furosemida por meio de métodos espectrofotométricos, por apresentarem boa sensibilidade e ter custos mais acessíveis. 0 presente trabalho teve como objetivo desenvolver e validar uma metodologia analítica para determinação deste fármaco em matéria-prima, utilizando a espectrofotometria de ultravioleta. O método empregado demonstrou ser simples, rápido, específico, linear, exato, preciso e robusto para ser executado na rotina de um laboratório de controle de qualidade, sendo uma alternativa aos procedimentos utilizados. Ao aplicar o método desenvolvido neste estudo para a análise de uma solução oral de furosemida constatouse necessidade e a importância de se validar o protocolo analítico de acordo com a formulação produzida.

PALAVRAS-CHAVE: furosemida, controle de qualidade, espectrofotometria, metodologia analítica, validação. 


\section{ABSTRACT:}

The furosemide is a drug that acts as diuretic been used to treat arterial hypertension. Among the methods used for their determination, there is the possibility of quantification of furosemide by spectrophotometrics methods, for presenting good sensitivity and have more affordable cost. This study aimed to develop and validate an analytical methodology for determination of this drug in raw material, using ultraviolet spectrophotometry. The method employed proved to be simple, rapid, specific, linear, accurate, precise and robust to run in a routine of a laboratory of quality control, being an alternative to procedures used. By applying the method developed in this study for the analysis of a oral solution of furosemide it was noted the need and importance of validating the analytical protocol in accordance with the formulation produced..

KEYWORDS: furosemide, quality control, spectrophotometry, analytical methodology, validation. 


\section{INTRODUÇÃO:}

A furosemida (Figura 1) é um
diurético de alça que atua
predominantemente no segmento
ascendente espesso da alça de Henle,
induzindo a secreção de água e eletrólitos.
É eliminado na urina tanto por filtração
glomerular quanto por secreção tubular e
uma pequena porção deste fármaco é
metabolizada no fígado e eliminada nas

fezes. Além de sua utilização nos estados edematosos habituais associados à insuficiência cardíaca congestiva, à cirrose ou às doenças renais, a furosemida pode ser administrada em casos de emergência, como edema pulmonar agudo, quando o rápido início de ação é essencial (WESTFAL, 2005). É também indicada para o tratamento de hipertensão arterial leve a moderada (LOPES \& MARTINELLI, 2006).

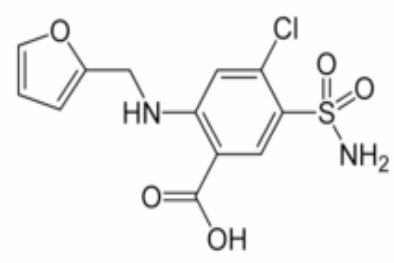

Figura 1 - Estrutura química da furosemida

O desenvolvimento de um novo método analítico deve garantir informações confiáveis e interpretáveis sobre a amostra e este deve ser submetido a uma avaliação denominada validação (RIBANI et al. 2004; LEITE, 1994). A validação de um novo método analítico deve garantir, por meio de estudos experimentais, que o método atenda às exigências das aplicações analíticas assegurando a confiabilidade dos resultados. Para tanto, deve apresentar especificidade, linearidade, intervalo, precisão, exatidão e robustez adequados à análise, conforme a finalidade do teste (BRASIL, 2003).

A monografia da furosemida matéria-prima - está presente na Farmacopéia Brasileira $4^{a}$ edição, $3^{\circ}$ fascículo (2001), na qual o doseamento é feito por titulação em meio não aquoso. Entretanto, este mesmo compêndio traz a monografia de comprimido de furosemida por um método espectrofotométrico. Considerando-se que o método titulométrico possui menor sensibilidade e pode sofrer maior número de interferências quando comparado ao método espectrofotométrico, o presente trabalho tem como objetivo aplicar e validar o método descrito para comprimidos na quantificação de furosemida - matéria-prima. Além disso, visou-se investigar a interferência que um método analítico pode sofrer devido a alterações nos componentes da formulação da solução oral. 


\section{MÉTODO:}

\section{Material e equipamento:}

Material:

Foram utilizados: substância química de referência furosemida (Farmacopéia Brasileira) - teor de 100,0\% - e matéria-prima de furosemida cedida por fornecedor idôneo, a qual foi submetida anteriormente a reações de identificação, como espectrofotometria no infravermelho e ponto de fusão. Os reagentes utilizados no estudo foram: hidróxido de sódio (Biotec) e água destilada. A vidraria utilizada foi calibrada pela Rede Brasileira de Calibração (RBC).

Equipamentos e condições analíticas:

Como pré-requisito para validação de métodos analíticos, os equipamentos e instrumentos foram previamente qualificados e certificados.

Para as leituras das absorbâncias, foram utilizados espectrofotômetros das marcas Cary 50 Bio (Varian) e B582 (Micronal), no comprimento de onda de $271 \mathrm{~nm}$. O ajuste do zero foi feito com solução de hidróxido de sódio $0,1 \mathrm{M}$.

Doseamento de Furosemida (matériaprima e solução oral):

- Preparo do padrão:

Pese o equivalente a $40 \mathrm{mg}(0,040 \mathrm{~g}) \mathrm{de}$ furosemida padrão, transfira para balão volumétrico de $100 \mathrm{~mL}$, complete o volume com $\mathrm{NaOH} 0,1 \mathrm{M}$, agite mecanicamente por 10 minutos. Pipete, volumetricamente, $1 \mathrm{~mL}$ desta solução para balão volumétrico de $50 \mathrm{~mL}$, solubilize e complete o volume com $\mathrm{NaOH}$ $0,1 \mathrm{M}$.

Concentração final do padrão: 8,0 x $10^{-6} \mathrm{~g} / \mathrm{mL}$.

- Preparo da amostra:

Transfira o equivalente a $100 \mathrm{mg}$ de furosemida para balão volumétrico de 250 $\mathrm{mL}$. Dissolva em $150 \mathrm{~mL}$ de $\mathrm{NaOH} \mathrm{0,1M}$, agite mecanicamente por 10 minutos. Complete o volume com $\mathrm{NaOH} 0,1 \mathrm{M}$, homogeneíze, filtre e dilua $5 \mathrm{~mL}$ do filtrado para $250 \mathrm{~mL}$ de $\mathrm{NaOH} 0,1 \mathrm{M}$, faça a leitura. Meça as absorvâncias das soluções resultantes em $271 \mathrm{~nm}$, utilizando $\mathrm{O} \mathrm{NaOH}$ $0,1 \mathrm{M}$ como branco.

$\rightarrow$ Cálculos:

$T=\underline{C p \times A a \times 100}$ Cta $\times$ Ap

Onde:

$\mathrm{T}=$ teor da amostra em porcentagem;

$\mathrm{Cp}=$ concentração do padrão $(\mathrm{mg} / \mathrm{mL})$;

$\mathrm{Aa}=$ absorbância da

amostra;

Cta $=$ concentração teórica da amostra;

Ap = absorbância do padrão. 
Estudo da validação:

Os parâmetros avaliados na validação do método para furosemida (matéria-prima) foram especificidade, linearidade, intervalo, precisão (repetibilidade e precisão intermediária), limite de detecção, limite de quantificação, exatidão e robustez.

- Especificidade: foi avaliada a partir da realização de varredura entre 200 e 800 nm do solvente, solução amostra e solução padrão, com a finalidade de garantir que o método analítico não sofre interferências de outros componentes que estarão presentes na amostra, tais como os solventes e impurezas.

- Linearidade: foi determinada pela realização da curva de calibração. A partir da solubilização da substância química de referência em solução de hidróxido de sódio $0,1 \mathrm{M}$, foram preparadas cinco soluções subseqüentes, utilizando o mesmo solvente, nas concentrações de 0,004; 0,006; 0,008; 0,01 e 0,012 $\mathrm{mg} / \mathrm{mL}$, dentro da faixa de 50-150\%. A curva de calibração foi preparada e analisada em duplicata. O critério mínimo aceitável do coeficiente de correlação (r) foi de 0,99 (BRASIL, 2003).

- Intervalo: foi determinado a partir do estudo de linearidade.

- Precisão: foi avaliada nos níveis: repetibilidade e precisão intermediária. A repetibilidade foi determinada pela análise de seis amostras individuais a $100 \%$. Para a precisão intermediária, foram testadas réplicas da concentração a $100 \%$ em dias diferentes e com analistas diferentes. Ambos os parâmetros foram expressos através do coeficiente de variação (CV) entre as amostras.

- Limite de deteç̧ão (LD) e quantificação (LQ): foram obtidos a partir de curva de calibração determinada em triplicata nas concentrações de 0,$5 ; 1,0 ; 2,5 ; 5,0$ e $10,0 \%$. LQ foi estabelecido por meio da equação: $L Q=D P a \times 10 / I C$;

em que: $D P a$ é o desvio padrão do intercepto com o eixo y de 3 curvas de calibração construídas contendo concentrações do fármaco próximas ao suposto limite de quantificação.

LD, foi determinado pela equação: $\mathrm{LD}=\mathrm{Dpa} \times 3 / \mathrm{IC}$;

em que: $\mathrm{DPa}$ é o desvio padrão do intercepto com o eixo y de 3 curvas de calibração construídas contendo concentrações do fármaco próximas ao suposto limite de quantificação.

- Exatidão: foi avaliada pelo teste de recuperação, analisando-se, em triplicata, amostras de concentração conhecida $(0,004 ; 0,008$ e $0,012 \mathrm{mg} / \mathrm{mL})$ equivalentes a 50,100 e $150 \%$, respectivamente, da concentração teórica analisada. 
- Robustez: a robustez do método avaliou as seguintes variáveis: comprimento de onda ( $\pm 2 \mathrm{~nm})$ e marca do espectrofotômetro.

Estudo da possibilidade de análise de uma solução oral de furosemida pelo método usado para matéria-prima:
$\mathrm{Na}$ investigação da aplicabilidade do método validado para matéria-prima ser empregado na análise de soluções de furosemida foram manipuladas duas formulações distintas, descritas no Quadro 1.

\begin{tabular}{|l|c|l|c|}
\hline \multicolumn{2}{|c|}{ Formulação 1: } & \multicolumn{2}{c|}{ Formulação 2: } \\
\hline Furosemida & $1,0 \mathrm{~g}$ & Furosemida & $1,0 \mathrm{~g}$ \\
\hline Etanol & $10 \% \mathrm{v} / \mathrm{v}$ & Etanol & $5 \% \mathrm{v} / \mathrm{v}$ \\
\hline Glicerina & $10 \% \mathrm{v} / \mathrm{v}$ & Glicerina & $10 \% \mathrm{v} / \mathrm{v}$ \\
\hline Hidróxido de sódio $20 \%$ & $\mathrm{pH} 8,0 \mathrm{a} 9,5$ & Hidróxido de sódio $20 \%$ & $\mathrm{pH} 8,0 \mathrm{a} 9,5$ \\
\hline EDTA-Na 2 & $0,1 \% \mathrm{p} / \mathrm{v}$ & EDTA-Na & $0,1 \% \mathrm{p} / \mathrm{v}$ \\
\hline Sulfito de sódio & $0,1 \% \mathrm{p} / \mathrm{v}$ & Metabissulfito de sódio & $0,1 \% \mathrm{p} / \mathrm{v}$ \\
\hline Metilparabeno & $0,005 \% \mathrm{p} / \mathrm{v}$ & Metilparabeno & $0,05 \% \mathrm{p} / \mathrm{v}$ \\
\hline Propilparabeno & $0,002 \% \mathrm{p} / \mathrm{v}$ & Propilparabeno & $0,02 \% \mathrm{p} / \mathrm{v}$ \\
\hline Sorbitol 70\% & $\mathrm{qsp}(100 \mathrm{~mL})$ & Sorbitol 70\% & $\mathrm{qsp}(100 \mathrm{~mL})$ \\
\hline
\end{tabular}

Quadro 1- Formulações de soluções orais de furosemida analisadas

Para ambas as formulações foram preparados, em paralelo, uma solução matriz (placebo) contendo apenas os excipientes. Os reagentes utilizados foram hidróxido de sódio (Biotec) e água destilada.

As soluções amostra, padrão e a matriz foram preparadas a partir da solubilização em hidróxido de sódio 0,1M, segundo metodologia adaptada da Farmacopéia Brasileira, 2001. As soluções resultantes foram submetidas à varredura em espectrofotômetro na faixa de 200 a $800 \mathrm{~nm}$. Construiu-se a partir da solução padrão uma curva de calibração na faixa de 50 a $150 \%$ da concentração de trabalho $(0,008 \mathrm{mg} / \mathrm{mL})$, a partir da qual calculouse a porcentagem de interferência da matriz.

\section{RESULTADOS:}

Dentre as atribuições de um laboratório de controle de qualidade destaca-se a garantia de que sejam feitas as validações necessárias, de procedimentos analíticos, calibração e qualificação dos equipamentos e vidrarias de precisão (LACHMAN et al., 2001; PINTO et al, 2003; CIRILO et al., 2007).

Neste estudo, comprovou-se a especificidade do método para análise da furosemida matéria-prima, uma vez que o mesmo não sofreu interferência do solvente utilizado no comprimento de onda de deteç̧ão $(271 \mathrm{~nm})$. Os espectros da varredura das soluções padrão e amostra foram idênticos, com pico em 271nm; já o espectro da varredura do solvente não apresentou nenhum pico de absorção.

Os resultados para o parâmetro de linearidade (Figura 2) demonstraram que o 
método apresenta intervalo linear na faixa de 50 a $150 \%$.

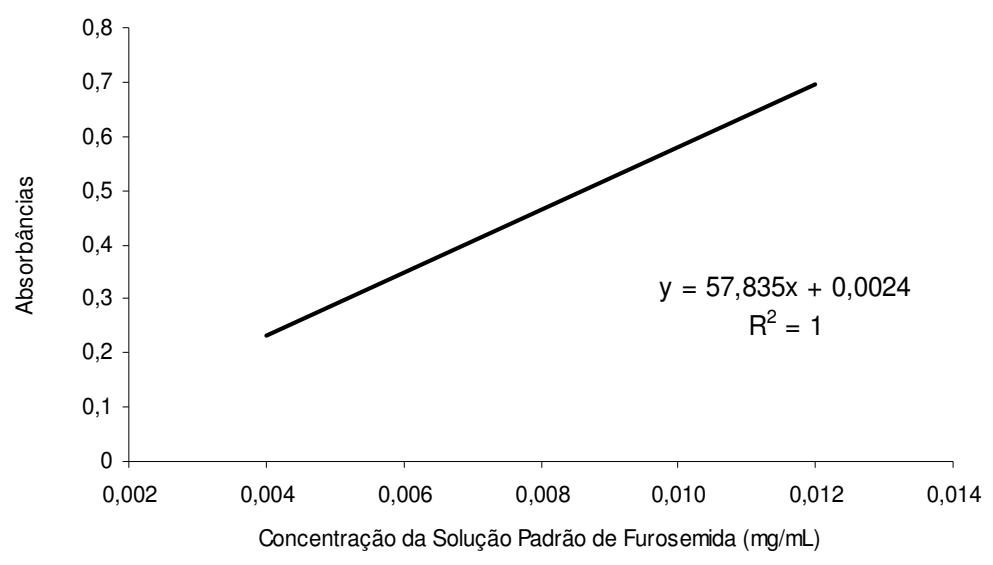

Figura 2 - Curva de calibração da Furosemida SQR.

O método apresentou-se preciso nos dois níveis avaliados (Tabelas 1 e 2), com coeficientes de variação (CV) inferiores ao especificado pela resolução vigente $(5,0 \%)$, dentro da faixa de concentração avaliada.

Tabela 1 - Resultados da precisão (repetibilidade)

\begin{tabular}{|c|c|c|c|c|c|}
\hline Determinações & $\begin{array}{l}\text { Massas } \\
\text { pesadas } \\
(\mathrm{mg})\end{array}$ & $\begin{array}{l}\text { Concentração } \\
\text { teórica da } \\
\text { amostra } \\
(\mathrm{mg} / \mathrm{mL})\end{array}$ & $\begin{array}{l}\text { Concentração } \\
\text { real da amostra } \\
(\mathrm{mg} / \mathrm{mL})\end{array}$ & $\begin{array}{c}\text { Recuperação } \\
(\%)\end{array}$ & Absorbâncias \\
\hline D_1 & 40,4 & 0,00808 & 0,00809 & 100,13 & 0,4703 \\
\hline D_2 & 40,8 & 0,00816 & 0,00804 & 98,53 & 0,4674 \\
\hline D_3 & 40,7 & 0,00814 & 0,00796 & 97,84 & 0,463 \\
\hline D_4 & 40,5 & 0,00810 & 0,00805 & 99,37 & 0,4679 \\
\hline D_5 & 40,9 & 0,00818 & 0,00807 & 98,61 & 0,4689 \\
\hline \multirow[t]{7}{*}{ D_6 } & 40,4 & 0,00808 & 0,00800 & 99,04 & 0,4652 \\
\hline & & & & Média & \\
\hline & & & & 98,92 & \\
\hline & & & & $\mathrm{DP}$ & \\
\hline & & & & 0,786102175 & \\
\hline & & & & DPR & \\
\hline & & & & 0,794703552 & \\
\hline
\end{tabular}

Tabela 2 - Resultados da precisão intermediária 


\begin{tabular}{|c|c|c|c|c|c|}
\hline Determinações & $\begin{array}{l}\text { Massas } \\
\text { pesadas } \\
(\mathrm{mg})\end{array}$ & $\begin{array}{c}\text { Concentração } \\
\text { teórica da } \\
\text { amostra } \\
\text { (mg/mL) }\end{array}$ & $\begin{array}{l}\text { Concentração } \\
\text { real da amostra } \\
(\mathrm{mg} / \mathrm{mL})\end{array}$ & Teor (\%) & Absorbâncias \\
\hline D_1 & 40,2 & 0,00804 & 0,00804 & 100,02 & 0,4675 \\
\hline D_2 & 40,4 & 0,00808 & 0,00811 & 100,41 & 0,4716 \\
\hline D_3 & 40,0 & 0,00800 & 0,00809 & 101,11 & 0,4702 \\
\hline D_4 & 40,1 & 0,00802 & 0,00804 & 100,25 & 0,4674 \\
\hline D_5 & 40,6 & 0,00812 & 0,00815 & 100,36 & 0,4737 \\
\hline \multirow[t]{7}{*}{ D_6 } & 40,5 & 0,00810 & 0,00811 & 100,11 & 0,4714 \\
\hline & & & & Média & \\
\hline & & & & 100,38 & \\
\hline & & & & $\mathrm{DP}$ & \\
\hline & & & & 0,385658241 & \\
\hline & & & & DPR & \\
\hline & & & & 0,384212534 & \\
\hline
\end{tabular}

A exatidão do método foi comprovada pelo estudo de três concentrações da amostra e os valores encontrados encontraram dentro da faixa de 97,51 a $100,28 \%$ (Tabela 3).

Tabela 3 - Resultados da exatidão

\begin{tabular}{|c|c|c|c|c|c|c|c|c|c|}
\hline $\begin{array}{l}\text { Determi- } \\
\text { nações }\end{array}$ & $\begin{array}{l}\text { Massas } \\
\text { pesadas } \\
(\mathrm{mg})\end{array}$ & $\begin{array}{c}\text { Concentração } \\
\text { teórica da } \\
\text { amostra } \\
(\mathrm{mg} / \mathrm{mL})\end{array}$ & $\begin{array}{c}\text { Concentração } \\
\text { real da } \\
\text { amostra } \\
(\mathrm{mg} / \mathrm{mL})\end{array}$ & $\begin{array}{l}\text { Recupe- } \\
\text { ração } \\
(\%)\end{array}$ & $\begin{array}{l}\text { Absorbân- } \\
\text { cias }\end{array}$ & $\begin{array}{c}\text { Média } \\
\text { Recuperação } \\
\text { (\%) }\end{array}$ & DP & DPR & $\begin{array}{c}\text { Recuperação } \\
(\mathrm{mg} / \mathrm{mL})\end{array}$ \\
\hline D_1 & 20,2 & 0,00404 & 0,00393 & 97,24 & 0,2296 & \multirow{4}{*}{97,5147} & \multirow{3}{*}{1,7200} & \multirow{3}{*}{1,76} & 0,003928417 \\
\hline D_2 & 20,1 & 0,00402 & 0,00399 & 99,36 & 0,2334 & & & & 0,003994121 \\
\hline D_3 & 20,3 & 0,00406 & 0,00390 & 95,95 & 0,2277 & & & & 0,003895565 \\
\hline D_4 & 40,8 & 0,00816 & 0,00803 & 98,36 & 0,4666 & & \multirow{3}{*}{0,6701} & \multirow{3}{*}{0,68} & 0,008026282 \\
\hline D_5 & 40,5 & 0,00810 & 0,00805 & 99,41 & 0,4681 & \multirow[t]{2}{*}{98,6448} & & & 0,008052218 \\
\hline D_6 & 40,9 & 0,00818 & 0,00803 & 98,16 & 0,4668 & & & & 0,00802974 \\
\hline D_7 & 60,1 & 0,01202 & 0,01207 & 100,45 & 0,7007 & \multirow{3}{*}{100,28} & \multirow{3}{*}{0,1478} & \multirow{3}{*}{0,15} & 0,012074004 \\
\hline D_8 & 60,2 & 0,01204 & 0,01206 & 100,17 & 0,6999 & & & & 0,012060171 \\
\hline D_9 & 60,3 & 0,01206 & 0,01209 & 100,23 & 0,7015 & & & & 0,012087836 \\
\hline
\end{tabular}

Os limites de detecção $\left(2,77 \times 10^{-5} \mathrm{mg} / \mathrm{mL}\right)$ e de quantificação $\left(9,24 \times 10^{-5} \mathrm{mg} / \mathrm{mL}\right)$ obtidos demonstram que o método é sensível. É importante salientar que esses parâmetros foram avaliados, embora não sejam obrigatórios para os testes enquadrados na Categoria I da Resolução 899/2003 (BRASIL, 2003).

Os resultados obtidos demonstram que o mesmo se apresentou robusto para variações de até 2 unidades no comprimento de onda e para variações da 
marca do espectrofotômetro (Tabela 4). dentro dos limites preconizados.

Os coeficientes de variação obtidos estão

Tabela 4 - Robustez com relação ao equipamento e aos comprimentos de onda empregados

\begin{tabular}{|c|c|c|c|c|c|c|c|}
\hline Parâmetros & & Abs1 & Abs2 & Abs3 & $\begin{array}{l}\text { Abs } \\
\text { média }\end{array}$ & $\begin{array}{l}\text { Concentra- } \\
\text { ção } \\
(\mathrm{mg} / \mathrm{mL})\end{array}$ & $\mathrm{CV}(\%)$ \\
\hline \multirow[b]{2}{*}{ Espectrofotômetro } & LCQM FF/UFG & 0,4637 & 0,4654 & 0,4640 & 0,4644 & 0,0080 & \multirow[b]{2}{*}{0,33} \\
\hline & $\begin{array}{l}\text { Lab. Enzimologia } \\
\text { FF/UFG }\end{array}$ & 0,4530 & 0,4520 & 0,4550 & 0,4533 & 0,0078 & \\
\hline \multirow{3}{*}{$\begin{array}{l}\text { Comprimento de } \\
\text { Onda }\end{array}$} & $271 \mathrm{~nm}$ & 0,4630 & 0,4642 & 0,4620 & 0,4631 & 0,0080 & \\
\hline & $269 \mathrm{~nm}$ & 0,4599 & 0,4599 & 0,4591 & 0,4596 & 0,0079 & 0,10 \\
\hline & $273 \mathrm{~nm}$ & 0,4457 & 0,4468 & 0,4460 & 0,4462 & 0,0077 & 0,12 \\
\hline
\end{tabular}

Abs = absorbância

Com a intenção de realizar uma investigação da aplicabilidade de uso do método validado para furosemida matériaprima na análise de um produto acabado (solução oral), iniciou-se com o ensaio de especificidade, que é um dos parâmetros relacionados à validação de produtos acabados e demonstra a capacidade que um método possui de medir exatamente um composto em presença de outros componentes, tais como impurezas, produtos de degradação e componentes da matriz (BRASIL, 2003).

A partir dos espectros de absorção das soluções amostra, padrão e matriz comprovou-se a interferência dos constituintes da formulação 2 na especificidade do método (Figuras 3 e 4). No espectro da Formulação 1 foram observados picos de absorbância no comprimento de onda de $271 \mathrm{~nm}$ para o padrão e a amostra, não sendo observado pico significativo na varredura da solução matriz (Figura 3). O espectro da Formulação 2 apresentou picos de absorbância em 271 nm para a solução amostra e padrão, entretanto a solução matriz apresentou pico em 294 nm, demonstrando a interferência dos componentes da formulação na especificidade do método (Figura 4).

Pode-se verificar na formulação 2 uma menor concentração de etanol, maior concentração de parabenos e a substituição de sulfito por metabissulfito de sódio (Figura 4). Não foi investigado qual (s) destes adjuvantes farmacotécnicos ocasiona $(m)$ a absorção em comprimento de onda próximo ao do princípio ativo (furosemida). 


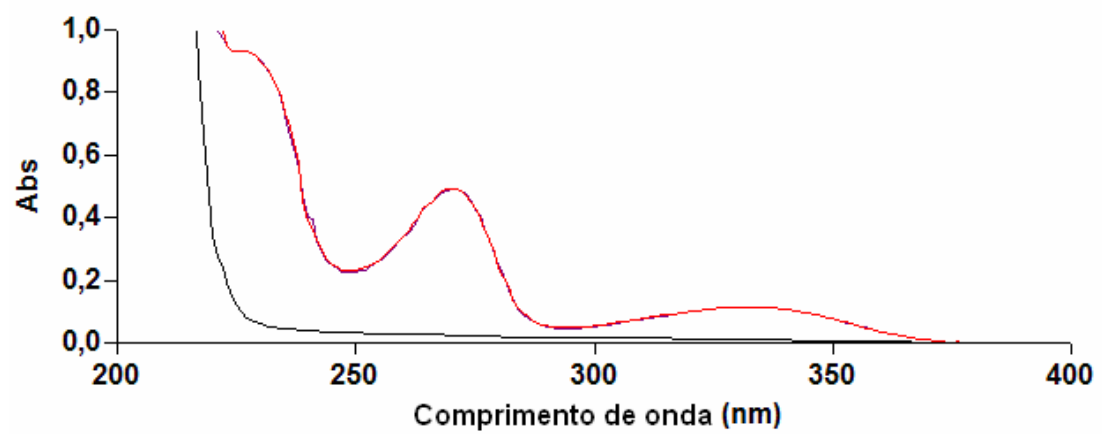

Figura 3 - Espectro de absorção da matriz (preto), amostra e padrão sobrepostos (vermelho).

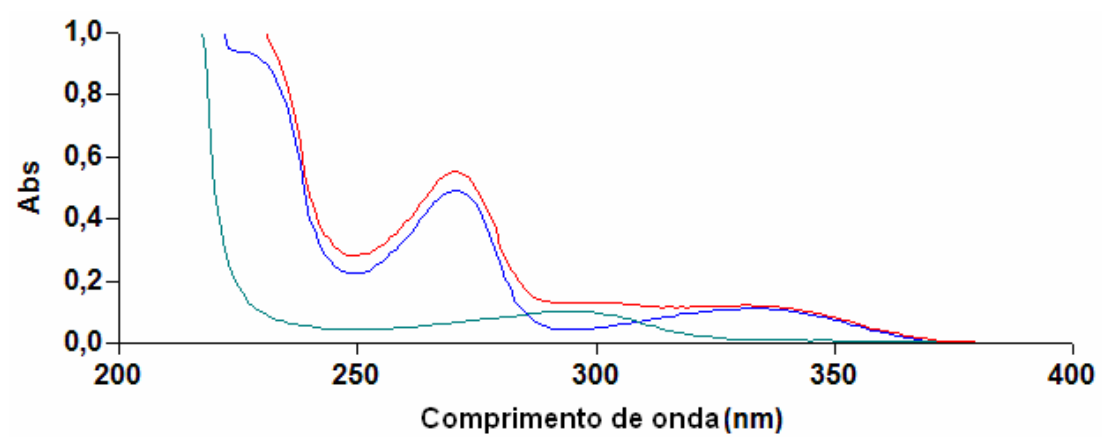

Figura 4 - Espectro de absorção da matriz (verde), amostra (vermelho) e padrão (azul).

A porcentagem de interferência da matriz da formulação 2 foi de 8,97\%. Este valor foi calculado através da equação da reta $y=57,835 x+0,0024$. Deste modo, demonstrou-se que a formulação 2 não pode ser analisada por este método espectrofotométrico. Pode-se empregar outro método, a ser desenvolvido, possivelmente por cromatografia líquida de alta eficiência para análise desta formulação ou alterar a formulação, por exemplo, usando a de número 1 , que não sofreu interferência conforme a matriz. Estes resultados reforçam a teoria de que um mesmo método analítico pode ser capaz de analisar a matéria-prima, porém não estar apto para a análise de formulações desta matéria-prima.

$$
\text { O método, quando aplicado na }
$$
análise da formulação 1 , mostrou ser preciso (Tabelas 5), entretanto não foi exato em relação ao DPR, ao se pesar uma massa de $50 \%$ (Tabela 6).

Tabela 5- Precisão intra-corrida/repetibilidade (solução oral de furosemida) 


\begin{tabular}{|c|c|c|c|c|c|}
\hline Determinações & $\begin{array}{l}\text { Massas } \\
\text { pesadas } \\
(\mathrm{mg})\end{array}$ & $\begin{array}{c}\text { Concentração } \\
\text { teórica da } \\
\text { amostra } \\
(\mathrm{mg} / \mathrm{mL})\end{array}$ & $\begin{array}{l}\text { Concentração } \\
\text { real da amostra } \\
(\mathrm{mg} / \mathrm{mL})\end{array}$ & $\begin{array}{c}\text { Recuperação } \\
(\%)\end{array}$ & Absorbâncias \\
\hline D_1 & 100 & 0,00800 & 0,00912 & 114,01 & 0,5299 \\
\hline D_2 & 100 & 0,00800 & 0,00908 & 113,53 & 0,5277 \\
\hline D_3 & 100 & 0,00800 & 0,00898 & 112,28 & 0,5219 \\
\hline D_4 & 100 & 0,00800 & 0,00863 & 107,87 & 0,5015 \\
\hline D_5 & 100 & 0,00800 & 0,00869 & 108,65 & 0,5051 \\
\hline \multirow[t]{7}{*}{ D_6 } & 100 & 0,00800 & 0,00907 & 113,40 & 0,5271 \\
\hline & & & & Média & \\
\hline & & & & 111,63 & \\
\hline & & & & $\mathrm{DP}$ & \\
\hline & & & & 2,678422371 & \\
\hline & & & & DPR & \\
\hline & & & & 2,399481986 & \\
\hline
\end{tabular}

Tabela 6 - Exatidão/recuperação (solução oral de furosemida)

\begin{tabular}{ccccccccc}
\hline $\begin{array}{c}\text { Massas } \\
\text { pesadas } \\
(\mathrm{mg})\end{array}$ & $\begin{array}{c}\text { Concentração } \\
\text { teórica da } \\
\text { amostra } \\
(\mathrm{mg} / \mathrm{mL})\end{array}$ & $\begin{array}{c}\text { Concentração } \\
\text { real da } \\
\text { amostra } \\
(\mathrm{mg} / \mathrm{mL})\end{array}$ & $\begin{array}{c}\text { Recupe- } \\
\text { ração } \\
(\%)\end{array}$ & $\begin{array}{c}\text { Absorbân- } \\
\text { cias }\end{array}$ & $\begin{array}{c}\text { Média } \\
\text { Recuperação } \\
(\%)\end{array}$ & DP & DPR & $\begin{array}{c}\text { Recuperação } \\
(\mathrm{mg} / \mathrm{mL})\end{array}$ \\
\hline 50 & 0,00400 & 0,00474 & 118,53 & 0,2766 & & & & 0,004741074 \\
50 & 0,00400 & 0,00366 & 91,38 & 0,2138 & 104,7376 & 13,5783 & 12,96 & 0,003655226 \\
50 & 0,00400 & 0,00417 & 104,31 & 0,2437 & & & & 0,004172214 \\
100 & 0,00800 & 0,00911 & 113,86 & 0,5292 & & & & 0,009108671 \\
100 & 0,00800 & 0,00914 & 114,25 & 0,531 & 114,3483 & 0,5473 & 0,48 & 0,009139794 \\
100 & 0,00800 & 0,00920 & 114,94 & 0,5342 & & & & 0,009195124 \\
150 & 0,01200 & 0,01288 & 107,37 & 0,7476 & & & & 0,012884931 \\
150 & 0,01200 & 0,01287 & 107,24 & 0,7467 & 107,51 & 0,3513 & 0,33 & 0,01286937 \\
150 & 0,01200 & 0,01295 & 107,91 & 0,7513 & & & & 0,012948906 \\
\hline
\end{tabular}

A robustez do método foi comprovada, os resultados demonstram que as variações de até 2 unidades no comprimento de onda e variações da marca do espectrofotômetro não interferem no método analítico.

\section{CONCLUSÃO:}

Os resultados da validação do método para determinação do teor de furosemida matéria-prima comprovaram que este é preciso, exato, linear, robusto e demonstrou especificidade. Portanto, demonstrou-se sua aplicabilidade na rotina de um laboratório de controle de qualidade. O método proposto neste trabalho representa uma alternativa para a quantificação de furosemida, uma vez que apresenta a confiabilidade requerida para um método analítico. 
Em relação à análise do produto acabado, o presente trabalho demonstrou a necessidade do desenvolvimento de métodos analíticos específicos para os diversos produtos acabados e assim, verifica-se a importância de se validar o método de acordo com a formulação desenvolvida. 


\section{REFERÊNCIAS BIBLIOGRÁFICAS:}

BRASIL. Ministério da Saúde. Agência Nacional de Vigilância Sanitária. Resolução 899, de 29 de maio de 2003. Guia para validação de métodos analíticos e bioanaliticos. Brasília, 2003.

WESTFALL, D.P. Drogas anti-hipertensivas. In CRAIG, C. R.; STITZEL, R. E. Farmacologia Moderna com Aplicações Clínicas. Rio de Janeiro: Editora Guanabara Koogan, 2005.

FARMACOPÉIA BRASILEIRA, São Paulo: Atheneu Editora, 2001.

CIRILO, H.N.C.; BARA, M.T.F.; GIL, E.S. Implantação do controle de qualidade. In GIL, E.S. Controle de qualidade fisico-químico de qualidade de medicamentos. São Paulo: Pharmabooks. 2007.

LACHMAN, L.; HANNA, S. A.; LIN, K. Controle e Garantia de Qualidade. In LACHMAN, L.; LIEBERMAN, H. A.; KANING, J. L. Teoria e Prática na Indústria Farmacêutica. v. II. Lisboa: Fundação Calouste Gulbenkian, 2001.

LEITE, F. Validação em análise química. Campinas: Editora Átomo, 2002.

PINTO, T. J. A.; KANECO, T. M.; OHARA, M. T. Controle Biológico de Qualidade de Produtos Farmacêuticos, Correlatos e Cosméticos. São Paulo: Editora Atheneu, 2003.

RIBANI, M. et al. Validação em métodos cromatográficos e eletroforéticos. Química Nova. v. 27, n. 5, p. 771- 780, 2004.

LOPES, A.A.; MARTINELLI, R. Farmacologia dos diuréticos. In SILVA, P. Farmacologia. Rio de Janeiro: Editora Guanabara Koogan, 2006. 